\title{
Social Media and Depression.
}

\author{
Thapa $\mathbf{R}^{1}$, Subedi $\mathbf{S}^{2}$ \\ 1. Consultant Psychiatrist, Neuro-Cardio Hospital, Biratnagar, Nepal 2. Associate Professor, \\ Department of Psychiatry, UCMS, Bhairahawa, Nepal
}

E-mail *Corresponding author : thaparanjan2@ gmail.com

\section{BACKGROUND}

Depression is highly prevalent and has the highest DALY among the illnesses. The prevalence of depression has been increasing recently. Multiple etiological factors has been found to contribute for the development of this illness. Recently there has been a growing interest in the potential influence of social media use on psychological well-being.

Social media can be defined as "a group of Internet based applications that allow the creation and exchange of user-generated content,". Social media has become an integral component of connecting with friends and family, sharing personal content, and obtaining news and entertainment. The use of social media sites such as Facebook and Twitter has particularly increased among young adults. About 90 percent of young adults in the US use social media, and most of users visit these sites at least once a day. Social Media has changed the way the current generation connects with their peers and views the world. They spend less time connecting with their peers in person and more time in connecting with them by social media.

\section{ROLE OF SOCIAL INTERACTION}

Human beings are social animals. Social interaction is important for human well being. Multiple studies prove that people thrive when they have strong positive relationships with other people. There are many proven benefits of strong social ties like, it lower risks of mental illnesses; higher likelihood of positive health behaviors; lower likelihood of negative health behaviors, such as excessive alcohol use; and overall lower risk of morbidity and mortality. ${ }^{1,2}$

\section{EFFECT OF ONLINE INTERACTION}

There is controversy as to whether social media use may exacerbate or alleviate conditions such as depression and anxiety. Engagement via platforms such as Facebook, Twitter, Instagram, Snapchat etc may provide opportunities for keeping in touch with family and friends as well as other social interactions that may increase social capital and alleviate depression and anxiety. Use of social media may facilitate forming connections among people with potentially stigmatizing health conditions, including depression and anxiety. Nabi et al. showed that having a higher number of friends on Facebook significantly predicted higher perceived social support, reduced stress, and increased overall well-being, although the interpretation of their results is limited by the cross-sectional nature of the study. ${ }^{3}$ Other investigators have also concluded that it may be the quality of social media use that is associated with mental health outcomes rather than the quantity of time spent on social media sites, such that when social media is being used to leverage social resources, its overall impact may be positive. 4,5 However, most large-scale empiric work in this area suggests associations between time spent on social media and increased symptoms of depression and anxiety and decline in subjective well-being (Kross et al., 2013; Lin et al., 2016; .Andreassen et al., 2016; Block et al., 2014; Woods \& Scott, 2016). 6,7,8

Lin et al studied 1,787 adults ages 19 to 32 about social media use and depression. Results showed that compared to those in the lowest quartile of total time per day spent on Social media, participants in the highest quartile had significantly increased odds of depression (AOR $=1.66)$. Compared with those in the lowest 
quartile, individuals in the highest quartile of Social media site visits per week had significantly increased odds of depression (AOR $=2.74$ ). So it was concluded that Social media use was significantly associated with increased depression. ${ }^{7}$

Primack et al assessed multiple social media (SM) platform use among 1787 U.S. young adults ages 19 to 32. Results showed that compared to those who used 0 to $2 \mathrm{SM}$ platforms, participants who used 7 to $11 \mathrm{SM}$ platforms had substantially higher odds of having increased levels of both depression (AOR: 3.0) and anxiety symptoms (AOR : 3.2). Associations were linear ( $p<0.001$ for all) and robust. So it was concluded that use of multiple social media platforms is independently associated with symptoms of depression and anxiety, even when controlling for overall time spent on social media. ${ }^{9}$

Holly Shakya and Nicolas Christakis assessed the associations of both online and offline social networks with several subjective measures of well-being. They used 3 waves $(2013,2014$, and 2015) of data from 5,208 subjects in the nationally representative Gallup Panel Social Network Study survey, including social network measures, in combination with objective measures of Facebook use. They investigated the associations of Facebook activity and real-world social network activity with self-reported physical health, self-reported mental health, selfreported life satisfaction, and body mass index. Results showed that overall, the use of Facebook was negatively associated with well-being. For example, a 1-standard-deviation increase in "likes clicked" "links clicked" or "status updates" was associated with a decrease of 5\%$8 \%$ of a standard deviation in self-reported mental health. These associations were robust to multivariate cross-sectional analyses, as well as to 2-wave prospective analyses. The negative associations of Facebook use were comparable to or greater in magnitude than the positive impact of offline interactions, which suggests a possible tradeoff between offline and online relationships. ${ }^{10}$

One reason the correlation seems more than coincidental is that an increase in depression occurred in tandem with the rise in smart phone use. A 2017 study of over half a million 8 to 12th graders found that the number exhibiting high levels of depressive symptoms increased by 33 percent between 2010 and 2015. In the same period, the suicide rate for girls in that age group increased by 65 percent. Smartphones were introduced in 2007, and by 2015 fully 92 percent of teens and young adults owned a smartphone. The rise in depressive symptoms correlates with smartphone adoption during that period, even when matched year by year. Over that same time period there was a sharp spike in reports of students seeking help at college and university counseling centers, principally for depression and anxiety. Visits jumped 30 percent between 2010 and 2015. ${ }^{11}$ One interesting study from Denmark showed that cessation of Facebook use improved mood but that effect was sustained for few weeks only.

\section{DOES SOCIAL MEDIA CAUSE DEPRESSION?}

These studies show a correlation, not causation. The directionality of this association is not clear. It may be that individuals with depression tend to use more social media. For example, individuals with underlying depression and anhedonia may be more drawn to social media interactions rather than face-to-face interactions. It may also be that those who use increased amounts of social media subsequently develop increased depression.

\section{HOW DO SOCIAL MEDIA MAKE US UNHAPPY?}

Use of social media may detract from face-to face relationships, reduce investment in meaningful activities, increase sedentary behavior by encouraging more screen time, lead to Internet addiction, and erode self-esteem through unfavorable social comparisons.

\section{Lack of face to face interaction}

Current generation spend much less time connecting with their peers in person and more time connecting through social media. The rise in depression is because the connections social media users form electronically are less emotionally satisfying, leaving them feeling socially isolated. The less you are connected with human beings in a deep, empathic way, the less you're really getting the benefits of a social interaction. Indeed, one exception to the depression correlation is girls who are high users of social media but also keep up a high 
level of face-to-face social interaction. One study showed that those girls who interact intensely offline as well as through social media don't show the increase in depressive symptoms than those who interact less in person do. More time spend on social media means less communication with family members which leads to deterioration in bond between them and misunderstandings which may lead to Depression.

\section{Social media and perceived isolation}

Social isolation is a state in which an individual lacks a sense of social belonging, true engagement with others, and fulfilling relationships. It has two components one objective and another subjective or perceived which are related but not the same. One national study of young adults by Primack et al showed correlation between the time spent on social media and perceived social isolation (PSI). ${ }^{12}$ Again directionality is not confirmed. There is fear of missing out (FOMO). FOMO is really the fear of not being connected to our social world, and that need to feel connected sometimes trumps whatever's going on in the actual situation we're in. The more we use social media, the less we think about being present in the moment." One is occupied with worrying why we weren't invited to a party we're seeing on Instagram, or making sure we don't miss a single post from a friend. One is always playing catch-up to endless online updates, prioritizing social interactions that aren't as emotionally rewarding and can actually make us feel more isolated.

\section{Social Media and self-esteem}

Because people tend to display the most positive aspects of their lives on social media, it is possible for an individual to believe that his or her own life compares negatively to the carefully constructed positive lives of friends displayed on social media. It has been seen that this can lead to lower self-esteem and to depression. 13,14 Another cause of increase in depression is the loss of self-esteem, especially in teenage girls, when they compare themselves negatively with artfully curated images of those who appear to be prettier, thinner, more popular and richer. Many girls are bombarded with their friends posting the most perfect pictures of themselves, or they're following celebrities who do a lot of Photoshopping and have makeup and hair teams. If that's their model for what is normal, it can be very hard on their selfconfidence. Indeed, image-driven Instagram shows up in surveys as the platform that most leads young people to report feeling anxiety, depression and worries about body image.

Curation of a perfect image may not only make others feel inadequate, it's unhealthy even for those who appear to be successful at it. People spend so much time on social media trying to post what they think the world will think is a perfect life. Look at how happy I am! Look how beautiful I am! Without that they're worried that their friends won't accept them. They're afraid of being rejected. And if they are getting positive feedback from their social accounts, they might worry that what their friends like isn't the "real" them.

\section{Less healthy activity}

Another source of depression may be what teenagers are not doing during while they're spending time on social media, including physical activity and things that generate a sense of accomplishment, like learning new skills and developing talents. If you're spending a lot of time on your phone, you have less time for activities that can build confidence, a sense of achievement and connectedness.

\section{Disrupted concentration}

Another thing disrupted by social media is the process of doing homework and other tasks that require concentration. It's common for teenagers to engage with friends on social media while studying. They take pride in being able to multitask, but evidence shows that it cuts down on learning and performance. Multitasking is generally not possible. What one ends up doing is really just switching back and forth between two tasks rather quickly. There is a cost to the brain. And with poorer concentration and constant interruption, homework takes substantially longer than it should, cutting into free time and adding to stress.

\section{Sleep deprivation and depression}

One of the important contributors to depression is sleep deprivation which can be caused, or exacerbated, by social media. Research shows that 60 percent of adolescents are looking at their phones in the last hour before sleep, and 
that they get on average an hour less sleep than their peers who don't use their phones before bed. ${ }^{15}$ Blue light from screens interferes with falling asleep and checking social media is not necessarily a relaxing or sleep-inducing activity.

\section{Cyberbullying}

According to Pew Research Institute four in ten Americans have experienced cyberbullying and almost all politicians have experienced it. It may also contribute to depression and anxiety.

\section{CONCLUSION:}

Relation between social media and depression is controversial. Many robust studies have clearly demonstrated clear relationship between social media and depression. Some investigators, however, have found no relationship between social media use and depression, and others suggested that social media use has a positive impact on well-being through increased social support and reinforcement of real-world relationships. However there is less reason to doubt that Social Media is causing Depression in at least some of our patients. While examining patients especially young adults and children we should also explore the impact of social media.

\section{REFERENCES:}

1. Kawachi I, Berkman LF. Social ties and mental health.J Urban Health. 2001;78(3):458-467.

2. Uchino BN. Social support and health: a review of physiological processes potentially underlying links to disease outcomes. J Behav Med. 2006;29(4):377-387

3. Nabi RL, Prestin A, So J. Facebook friends with (health) benefits? Exploring social network site use and perceptions of social support, stress, and well-being. Cyberpsychol Behav Socl Netw. 2013;16(10):721-727.

4. Feinstein BA, Hershenberg $R$, Bhatia $V$, et al. Negative social comparison on Facebook and depressive symptoms: rumination as a mechanism. Psychol Pop Media Cult. 2013; 2(3):161-170.

5. Vogel EA, Rose JP, Okdie BM, et al. Who compares and despairs? The effect of social comparison orientation on social media use and its outcomes. Pers Individ Dif. 2015;86: 249-256.

6. Kross E, Verduyn P, Demiralp E, et al. Facebook use predicts declines in subjective well-being in young adults. PLoS One [Internet] 2013 Jan;8(8):e69841.

7. Lin LY, Sidani JE, Shensa A, et al. Association between Social Media Use and Depression among U.S. Young Adults. Depress Anxiety 2016 Apr 33(4):323-331

8. Andreassen, C. S., Billieux, J., Griffiths, M. D., Kuss, D. J., Demetrovics, Z., Mazzoni, E., E Pallesen, S. (2016). The relationship between addictive use of social media and video games and symptoms of psychiatric disorders: A large-scale cross-sectional study. Psychology of Addictive Behaviors, 30, 252-262.

9. Primack BA, Shensa $A$, et al. Use of multiple social media platforms and symptoms of depression and anxiety: A nationally-representative study among U.S. young adults. Computers in Human Behavior 2017 Apr; 69: 1-9.

10. Shakya, H. B., E Christakis, N. A. (2017). Association of Facebook use with compromised well-being: $A$ longitudinal study. American Journal of Epidemiology, 185(3), 203-211.

11. Twenge JM., Joiner TE, et al. Increases in Depressive Symptoms, Suicide-Related Outcomes, and Suicide Rates Among U.S. Adolescents After 2010 and Links to Increased New Media Screen Time. Clinical Psychological Science 2017;6(1):3-17

12. Primack BA, Shensa A, et al. Social media Use and Perceived Social Isolation among young adults in the US. Am J Prev Med. 2017 Jul; 53(1): 1-8.

13. Chou HT, Edge N. "They are happier and having better lives than I am": the impact of using Facebook on perceptions of others' lives. Cyberpsychol Behav Socl Netw. 2012;15(2): 117-121.

14. Appel H, Gerlach AL, Crusius J. The interplay between Facebook use, social comparison, envy, and depression. Curr Opin Psychol. 2016;9:44-49.

15. Fyller C, Lehman E. Bedtime Use of Technology and Associated Sleep Problems in Children. Global Pediatric Health 2017; 4: 1-8. 